\title{
The Relationship Between Big Five Personality and Social Well-Being of Chinese Residents: The Mediating Effect of Social Support
}

\author{
Yanghang $\mathrm{Yu}^{1+}$, Yuanyuan $\mathrm{Zhao}^{2 \dagger}$, Dongyan $\mathrm{Li}^{1}$, Jingqiu Zhang ${ }^{3}$ and Jiewei $\mathrm{Li}^{4 *}$ \\ ${ }^{1}$ School of Public Finance and Management, Yunnan University of Finance and Economics, Kunming, China, ${ }^{2}$ Tourism and \\ Cultural Industry Research Institute, Yunnan University of Finance and Economics, Kunming, China, ${ }^{3}$ International College, \\ National Institute of Development Administration, Bangkok, Thailand, ${ }^{4}$ National Centre for Borderlands Ethnic Studies in \\ Southwest China at Yunnan University (NaCBES), Yunnan University, Kunming, China
}

OPEN ACCESS

Edited by:

Dario Monzani

University of Milan, Italy

Reviewed by:

Guangzeng Liu,

Southwest University, China

Carlos Salavera,

University of Zaragoza, Spain

*Correspondence:

Jiewei Li

191534248@qq.com

†These authors share first authorship

Specialty section:

This article was submitted to Personality and Social Psychology, a section of the journal

Frontiers in Psychology

Received: 03 October 2020

Accepted: 31 December 2020

Published: 05 March 2021

Citation:

Yu Y, Zhao Y, Li D, Zhang J and Li J (2021) The Relationship Between

Big Five Personality and Social Well-Being of Chinese Residents:

The Mediating Effect of Social

Support. Front. Psychol. 11:613659. doi: 10.3389/fpsyg.2020.613659
Previous studies have noted that personality traits are important predictors of well-being, but how big five personality influences social well-being is still unknown. This study aims to examine the link between big five personality and five dimensions of social well-being in the Chinese cultural context and whether social support can play the mediating effect in the process. This study included 1,658 participants from different communities in China, and regression analyses were conducted. Results revealed that five personality traits were significantly related to overall social well-being; extraversion was significantly related to social integration; agreeableness was positively related to all five dimensions of social well-being; conscientiousness was positively related to social actualization, social coherence, and social contribution; neuroticism was negatively related to social integration, social acceptance, social actualization, and social coherence; openness was positively related to social integration, social acceptance, social coherence, and social contribution. Social support plays mediating roles in the relationships between extraversion/agreeableness/conscientiousness/neuroticism/openness and social wellbeing, respectively.

Keywords: big five personality, social support, social well-being, China, mediating effect

\section{INTRODUCTION}

Personality variables are strong predictors of well-being, a large body of research has explored the associations between big five personality and subjective well-being (DeNeve and Cooper, 1998; Gutiérrez et al., 2005). Unfortunately, the psychological construct of well-being portrays adult wellbeing as a primarily private phenomenon largely neglecting individuals' social lives (Keyes, 2002; Hill et al., 2012). Individuals are embedded in social structures and communities; as such, it is necessary to evaluate one's circumstance and functioning in a society; more attention needs to be devoted on the topic of social well-being (Keyes, 1998). Previous studies focused on the social wellbeing from the perspective of interpersonal factors, such as sense of community (Sohi et al., 2017), and civic engagement (Albanesi et al., 2010). However, less work has examined social well-being from the level of the individual (Keyes and Shapiro, 2004).

Although there are few studies focusing on the relationship between five personality traits and social well-being (Hill et al., 2012; Joshanloo et al., 2012), their data come from United States or Iran; Chinese cultural background has been conducted to a lesser extent. Different countries have 
different cultural traditions. Personality is created through the process of enculturation (Hofstede and McCrae, 2004). The interplay of personality and cultural factors was found to predict residents' well-being significantly (Diener and Diener, 1995). Confucius culture has embedded itself in the daily life of the Chinese, however, studies about the relationship between personality and social well-being under the context of Chinese culture are largely overlooked.

In addition, present studies (Hill et al., 2012; Joshanloo et al., 2012) examine only the direct effect of personality on social well-being. The mechanism between big five personality and five dimensions of social well-being has been neglected. Additionally, social support can help individuals protect against the health consequences of life stress and increase their wellbeing (Cobb, 1976; Siedlecki et al., 2014). Thus, following a social support perspective, the present study examined not only the relationship between five personality traits and domains of social well-being, but also whether social support can play a mediating effect in the relationship between big five personality and social well-being.

\section{LITERATURE REVIEW AND HYPOTHESIS}

\section{Big Five Personality and Social Well-Being}

The big five personality consists of five general traits: extraversion, neuroticism, openness, agreeableness, and conscientiousness (John and Srivastava, 1999). Extraversion refers to the degree to which one is energetic, social, talkative, and gregarious. Agreeableness reflects the extent to which one is warm, caring, supportive, and cooperative and gets along well with others. Conscientiousness involves the extent to which one is well-organized, responsible, punctual, achievement-oriented, and dependable. Neuroticism means the degree to which one is worry, anxious, impulsive, and insecure. Openness reflects the degree to which one is imaginative, creative, curious, and broad-minded (Barrick et al., 2001; Funder and Fast, 2010). Many scholars assessed personality under different culture context by a combined emic-etic approach (John and Srivastava, 1999; Cheung et al., 2001). Even if there were researches that demonstrated several unique dimensions of personality under the Chinese culture (Cheung et al., 2001; Cheung, 2004), the generalizability of the big five trait taxonomy in China is still confirmed (Li and Chen, 2015; Minkov et al., 2019). Previous studies have consistently demonstrated that the big five are associated with subjective well-being (DeNeve and Cooper, 1998; Gutiérrez et al., 2005), however, the findings are mixed under different cultural context. For instance, Ha and Kim (2013) found openness has a positive effect on subjective well-being in South Korea residents, whereas another study by Hayes and Joseph (2003) in England found that openness was not associated with each of the three measures of subjective well-being.

Culture variables can explain differences in mean levels of well-being (Diener et al., 2003). With the uniqueness of Confucian cultural tradition and social setting, it is noteworthy to discuss the relationship between personality and well-being in Chinese cultural background, especially social-well-being.

Individuals are embedded in social structures. They need to face social challenges and evaluate their life quality and personal functioning by comparison to social criteria (Keyes and Shapiro, 2004). However, the research about social wellbeing has been almost completely neglected in the hedonic and psychological well-being models (Keyes, 2002; Joshanloo et al., 2012). Keyes (1998) proposed social well-being, which indicates to what degree individuals are functioning well in the social world they are embedded in. Social well-being can be described on multiple dimensions, including social integration, social contribution, social acceptance, social coherence, and social actualization. Social integration is the extent to which people feel commonality and connectedness to their neighborhood, community, and society. Social contribution refers to a value evaluation that one can provide to the society. Social acceptance entails a positive view of human nature and believes that people are kind. Social coherence refers to the perception of the quality and operation of the social world and reflects a belief that society is meaningful. Social actualization is the evolution of the potential and of society and includes a sense that social potentials can be realized through its institutions and citizens. In summary, social well-being emphasizes individuals' perceptions of and attitudes toward the whole society. Prior studies have found the effect of sense of community (Sohi et al., 2017), and social participation (Albanesi et al., 2010) on social well-being, Also, some studies have shown the outcomes of social well-being, such as anxiety problems (Keyes, 2005), general mental and physical health (Zhang et al., 2011), and prosocial behaviors (Keyes and Ryff, 1998). Personality traits and cultural factors are important predictors of wellbeing (Diener et al., 2003). However, the only studies about personality and social well-being were conducted in Iran or United States. It is still not known whether the association would be similar in a different cultural context (Hill et al., 2012; Joshanloo et al., 2012). For example, with the data from the MIDUS sample, Hill et al. (2012) found social well-being is positively related to extraversion, agreeableness, conscientiousness, emotional stability, and openness. In addition, previous studies did not test the correlation between five personality traits and five domains of social well-being entirely (Joshanloo et al., 2012). Personality shapes many of the attitudes and behaviors that form Keyes' different dimensions of social well-being. Thus, certain personalities would predict social wellbeing; for example, extraverted persons should be more socially integrated, whereas agreeable individuals should possess higher levels of social acceptance. Based on the above, we hypothesize the following:

Hypothesis $1_{\mathrm{a}}$ : Extraversion is positively related to social wellbeing.

Hypothesis $1_{\mathrm{b}}$ : Agreeableness is positively related to social well-being.

Hypothesis $1_{c}$ : Conscientiousness is positively related to social well-being. 
Hypothesis $1_{\mathrm{d}}$ : Neuroticism is negatively related to social well-being.

Hypothesis $1_{\mathrm{e}}$ : Openness is positively related to social wellbeing.

\section{The Mediating Effect of Social Support}

Social support refers to individuals' psychological or material resources from their own social networks that can assist them to cope with stressful challenges in daily lives (Cohen, 2004). It comes from a variety of sources, such as friends, family, and significant others (Taylor, 2011). Social support comprised both received and perceived social support (Oh et al., 2014; Hartley and Coffee, 2019). However, many studies showed that perceived social support is more effective at predicting residents' mental health than the received social support (Cohen and Syme, 1985). Perceived social support indicates recipients' perceptions concerning the general availability of support (Sarason et al., 1990), which fosters a sense of social connectedness in a network and provides resources with which to overcome obstacles in their lives (Lee et al., 2001; Chen, 2013). Social support theory emphasizes that social support is an important resource that can help individuals protect against life stress and increase their quality of lives (Cobb, 1976; Cohen and Wills, 1985). Numerous studies have explored the associations between social support and well-being, including subjective well-being (Brannan et al., 2013; Siedlecki et al., 2014) and psychological well-being (JasinskajaLahti et al., 2006; Wong et al., 2007). Although Inoue et al. (2015) found social support mediated the effect of team identification on community coherence, little research has addressed the effect of social support on social well-being. The benefits of social support come into play when individuals have to deal with social challenges and problems. Individuals with high level of social supports will better face social tasks (Cox, 2000). Harmonious social relationships can help residents to satisfy their social needs, better understand, and be confident of the social world. Therefore, their social well-being will increase.

Personality traits are stable predictors of social support (Swickert et al., 2010; Udayar et al., 2018; Barańczuk, 2019). Big five personality traits are found to be related to social support. Individuals with high levels of neuroticism report greater vulnerability to stress and negative affectivity, which could decrease the availability of social support (Ayub, 2015). Individuals who score high on extraversion always seek social interactions and tend to be cheerful and friendly. The positive emotions could increase their social support (Swickert et al., 2010). Individuals with high openness to experience are characterized by greater openness to emotions, appreciation of art and beauty, intellect, and liberalism. These characteristics would be significantly related to social support (Barańczuk, 2019). Agreeableness characteristics, such as modesty, compliance, and trust, may facilitate individuals building a more extensive social support network (Barańczuk, 2019). Conscientiousness are characterized by achievementstriving, self-discipline, orderliness, and dutifulness. These tendencies can help individuals better cope with life stress, so it is positively related to social support (Ayub, 2015). Culture is an important moderator between big five personality traits and social support association, but it has been largely overlooked in previous studies (Barańczuk, 2019). Therefore, studies about the relationship between five personality traits and social support under Chinese background are needed.

Previous studies discuss only the direct effect of personality on social well-being, but it remains unknown what mechanism(s) may explain this relation. Social support plays an important stress-buffering role when individuals are under high levels of life stress (Cohen, 2004). Individuals with different levels of personality traits (extraversion, agreeableness, conscientiousness, neuroticism, openness) will form different types of social support network. Further, social support will help individuals cope with social challenges and increase their social well-being. Based on the above, we hypothesize the following:

Hypothesis $2_{\mathrm{a}}$ : Social support mediates the relationship between extraversion and social well-being.

Hypothesis $2_{\mathrm{b}}$ : Social support mediates the relationship between agreeableness and social well-being.

Hypothesis $2_{\mathrm{c}}$ : Social support mediates the relationship between conscientiousness and social well-being.

Hypothesis $2_{\mathrm{d}}$ : Social support mediates the relationship between neuroticism and social well-being.

Hypothesis $2_{\mathrm{e}}$ : Social support mediates the relationship between openness and social well-being.

\section{MATERIALS AND METHODS}

\section{Participants and Procedure}

Community residents from five different districts in Kunming, Yunnan Province, were selected as participants by stratified random sampling technique. Four hundred questionnaires were distributed to each district. Participants would complete the questionnaires in a face-to-face interaction with an enumerator who helped them to answer the questionnaire that was in paper format. When we administered the survey, we emphasized that the data were collected for research purposes. Participants were encouraged to answer all the questions honestly and were reminded that their responses would be anonymous. Upon completion of answering the questionnaire, participants received a small gift (e.g., tissue) as compensation for their participation. A total of 2,000 questionnaires were distributed, and 1,721 responded. After dropping incomplete and invalid data, 1,658 respondents remained. The final sample consisted of 932 females $(56.2 \%)$ and 726 males $(43.8 \%)$, aged $18-81$ years (mean $=30.73$ years, $\mathrm{SD}=11.98$ years).

\section{Measures}

\section{Big Five Personality}

The 44-item Big Five Inventory (BFI; John et al., 1991) was used to measure the five broad personality traits. All items were evaluated on a 5-point Likert scale, ranging from "strongly disagree" to "strongly agree." Coefficient $\alpha$ reliabilities for the five trait scales in the present study were 0.707 for extraversion, 0.712 for agreeableness, 0.729 for conscientiousness, 0.706 for neuroticism, and 0.733 for openness. The Chinese version of BFI 
we used had been translated from English using common backtranslation procedures (Brislin, 1970; Li and Chen, 2015), and the validity had been conformed in previous studies (Zhou, 2010; Li and Chen, 2015).

\section{Social Support}

Participants rated their social support from Chen and Yu (2019) using scales that ranged from 1 (strongly disagree) to 5 (strongly agree). The measure comprised three items, such as "It is easy for me to find someone to help when I meet with difficulties." The entire survey demonstrated good reliability $(\alpha=0.733)$.

\section{Social Well-Being}

Social well-being was measured through Keyes's (1998) 15-item scale composed of five dimensions: social actualization, social integration, social acceptance, social contribution, and social coherence. Responses to this measure were assessed on a 5-point scale, from "strongly disagree" to "strongly agree." An example of measure items was "I believe that people are kind." The reliabilities of five dimensions were good (ranging from 0.702 to 0.725 ), and overall $\alpha$ reliability for the present sample was 0.791 . Previous studies had confirmed the validity of social well-being measurement of Chinese version we used (Miao and Wang, 2009; Chen and Yu, 2019; Chen et al., 2020).

\section{RESULTS}

\section{The Common Method Bias Examination}

As one of the main sources of measurement error, common method variance is a potential problem, which may be a threat to the validity of the conclusions. We tested for common method bias with a single-factor measurement model by combining all items into a single factor (Podsakoff et al., 2003; Rhee et al., 2017). Results showed a poor model fit [Comparative Fit Index $(\mathrm{CFI})=0.763$, Tucker-Lewis Index $(\mathrm{TLI})=0.695$, Goodness-of-Fit Index (GFI) $=0.719$, Root Mean square Residual $(\mathrm{RMR})=0.025$, Root Mean Square Error of Approximation $($ RMSEA $)=0.109]$. The above results suggested that there was no common method bias effect.

\section{Descriptive Statistics and Correlations Between the Study Variables}

There is no significant difference between the five different districts in Kunming. The correlation coefficients, means, and standard deviations are shown in Table 1. All the big five personality traits were correlated significantly with social support and five domains of social well-being (expect agreeableness and social coherence). Extraversion, agreeableness, conscientiousness, and openness were correlated positively with domains of social well-being (expect agreeableness and social coherence) and social support, whereas neuroticism correlated negatively with domains of social well-being and social support.

\section{Regression Analyses}

Statistical analyses were conducted with the Statistical Package for Social Sciences (SPSS, version 22.0). Based on preliminary analyses, multiple regression analyses were conducted to assess the relationship between the big five personality domains and dimensions of social well-being. Both gender and age were statistically controlled during the regression analysis, because there is evidence to show that social well-being likely increases with one's age (Chen and Li, 2014) and that men generally score higher on well-being than women do (Miao and Wang, 2009). OLS regression was used to test the hypothesis. In each regression analysis, one social well-being dimension was entered as the dependent variable; gender, age, and all five personality domains were entered as potential predictors. Results of the regression analyses are presented in Table 2. Five personality traits were significant predictors of overall social well-being. Extraversion $(\beta=0.052, p \leq 0.05)$, agreeableness $(\beta=0.197, p \leq 0.001)$, conscientiousness $(\beta=0.138, p \leq 0.001)$, and openness $(\beta=0.156$, $p \leq 0.001)$ are positively related to social well-being, whereas neuroticism $(\beta=-0.171, p \leq 0.001)$ is negatively related to social well-being. $\mathrm{H}_{\mathrm{a}}, \mathrm{H} 1_{\mathrm{b}}, \mathrm{H} 1_{\mathrm{c}}, \mathrm{H} 1_{\mathrm{d}}$, and $\mathrm{H} 1_{\mathrm{e}}$ are supported. Extraversion $(\beta=0.118, p \leq 0.001)$, agreeableness $(\beta=0.162$, $p \leq 0.001)$, neuroticism $(\beta=-0.065, p \leq 0.05)$, and openness $(\beta=0.086, p \leq 0.001)$ were significant predictors of social integration. Agreeableness $(\beta=0.268, p \leq 0.001)$, neuroticism $(\beta=-0.102, p \leq 0.001)$, and openness $(\beta=0.089, p \leq 0.001)$ were significantly associated with social acceptance. Agreeableness $(\beta=0.168, p \leq 0.001)$, conscientiousness $(\beta=0.111, p \leq 0.001)$, and neuroticism $(\beta=-0.110, p \leq 0.001)$ predicted social actualization significantly. Agreeableness $(\beta=-0.088, p \leq 0.001)$, conscientiousness $(\beta=0.060, p \leq 0.05)$, neuroticism $(\beta=-0.241$, $p \leq 0.001)$, and openness $(\beta=0.125, p \leq 0.001)$ were found to be predicting social coherence. Agreeableness $(\beta=0.120$, $p \leq 0.001)$, conscientiousness $(\beta=0.191, p \leq 0.001)$, and openness $(\beta=0.164, p \leq 0.001)$ were found to be predictors of social contribution.

\section{Mediation Analyses}

Further, mediation analysis was performed to determine whether the effect of big five personality on social wellbeing was mediated by social support. Mediation analyses were conducted following the recommendations of Preacher and Hayes (2004), using the PROCESS macro (version 3.0), developed by Hayes (2013). The current study used 5,000 bootstrapped samples with a $95 \%$ confidence interval. The results of this analysis are shown in Table 3. The results suggested five personality traits are related to social support significantly, and social support is positively related to social well-being. In addition, social support mediated the relationship between five personality traits and social well-being. $\mathrm{H} 2_{\mathrm{a}}, \mathrm{H} 2_{\mathrm{b}}, \mathrm{H} 2_{\mathrm{c}}, \mathrm{H} 2_{\mathrm{d}}$, and $\mathrm{H} 2_{\mathrm{e}}$ are supported.

\section{DISCUSSION AND CONCLUSION}

The results obtained from the survey of 1,658 Chinese residents demonstrated the effects of five personality traits on five dimensions of social well-being and the mediating role of social support in the associations between big five personality and social well-being. 
TABLE 1 | Correlations, means, and standard deviations of all study variables.

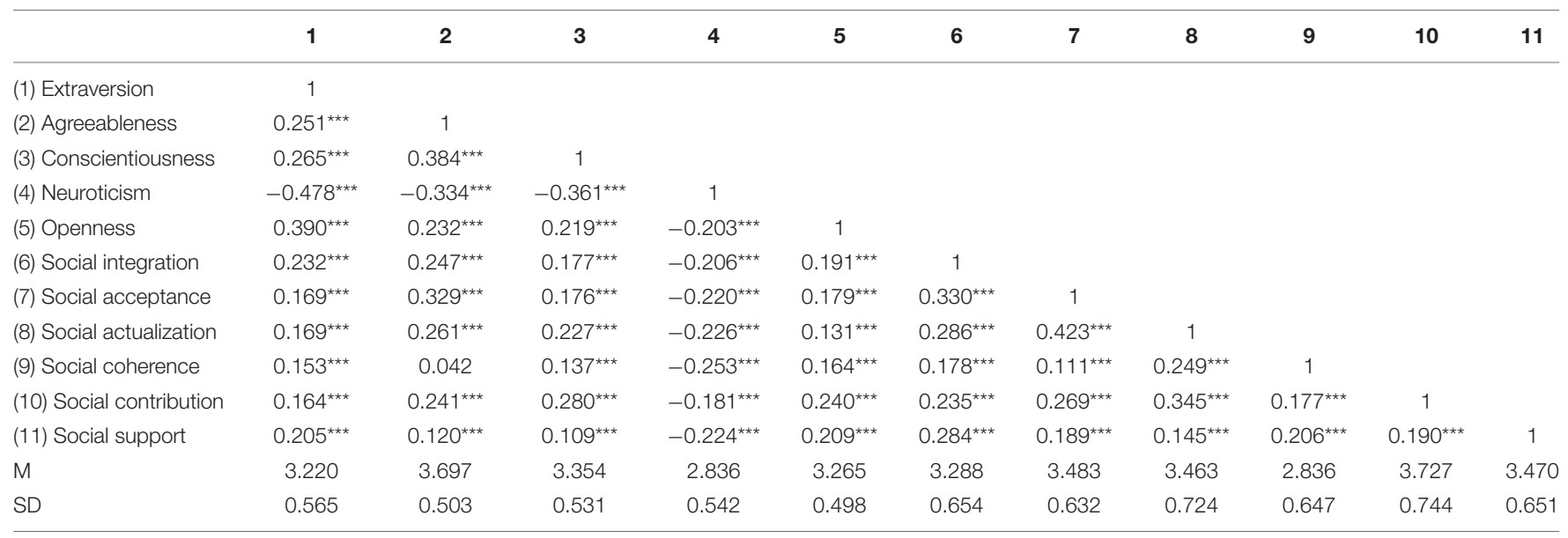

${ }^{* * *} p \leq 0.001,{ }^{* *} p \leq 0.01,{ }^{*} p \leq 0.05$.

TABLE 2 | Results of regression analyses for five personality traits predicting dimensions of social well-being.

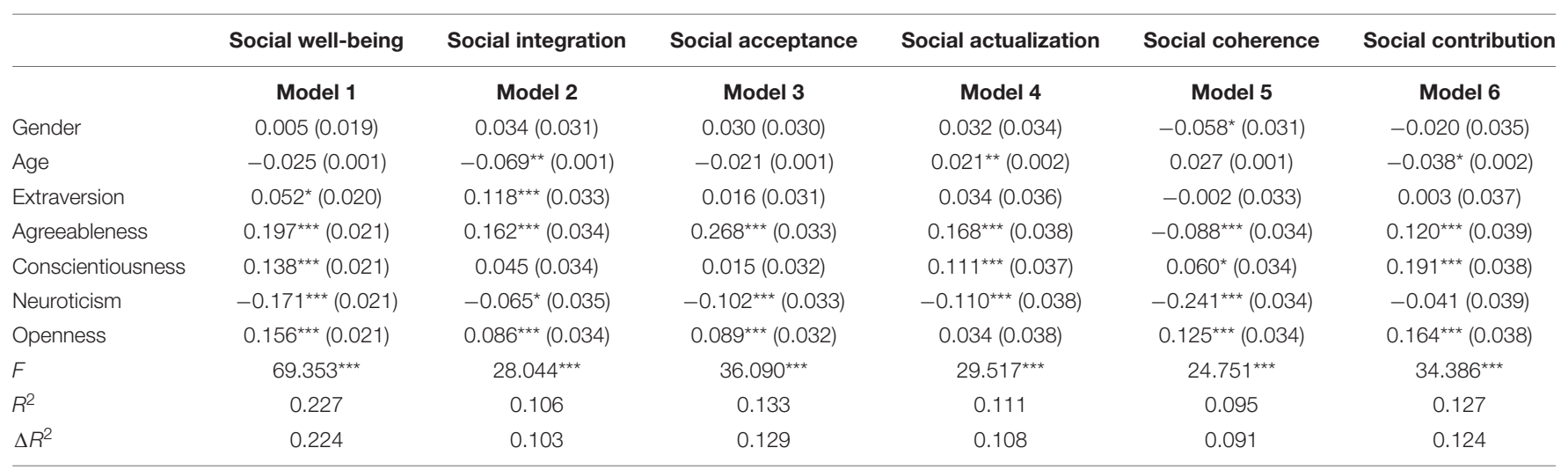

Coefficients are standardized betas. ${ }^{* * *} p \leq 0.001,{ }^{* *} p \leq 0.01,{ }^{*} p \leq 0.05$.

TABLE 3 | Summary of mediation analyses on five personality traits and social well-being (5,000 bootstraps).

\begin{tabular}{|c|c|c|c|c|c|c|c|c|}
\hline $\begin{array}{l}\text { Independent } \\
\text { variable }\end{array}$ & $\begin{array}{l}\text { Dependent } \\
\text { variable }\end{array}$ & c & $c^{\prime}$ & $a$ & $b$ & $a * b$ & Lower & Upper \\
\hline Extraversion & Social well-being & $0.215^{\star \star \star}(0.018)$ & $0.176^{\star \star \star}(0.018)$ & $0.224^{\star \star \star}(0.028)$ & $0.175^{\star \star \star}(0.016)$ & $0.039^{\star \star \star}(0.006)$ & 0.027 & 0.052 \\
\hline Conscientiousness & Social well-being & $0.261^{\star \star \star}(0.019)$ & $0.242^{\star \star \star}(0.018)$ & $0.096^{\star \star}(0.030)$ & $0.189^{\star \star \star}(0.015)$ & $0.018^{\star \star}(0.006)$ & 0.008 & 0.029 \\
\hline Neuroticism & Social well-being & $-0.272^{\star \star \star}(0.019)$ & $-0.232^{\star \star \star}(0.018)$ & $-0.238^{\star \star \star}(0.029)$ & $0.166^{\star \star \star}(0.015)$ & $-0.040^{\star \star \star}(0.006)$ & -0.054 & -0.029 \\
\hline Openness & Social well-being & $0.238^{\star \star \star}(0.021)$ & $0.189^{\star \star \star}(0.020)$ & $0.287^{\star \star \star}(0.031)$ & $0.173^{\star \star \star}(0.016)$ & $0.050^{\star \star \star}(0.007)$ & 0.036 & 0.065 \\
\hline
\end{tabular}

c: Total effect; c': direct effect; a: path from independent to mediator variable; b: path from mediator to dependent variable; $a$ * b: indirect effect; indirect effect; lower/upper: the $95 \%$ confidence interval of the indirect effect.

${ }^{* * *} p \leq 0.001,{ }^{* *} p \leq 0.01,{ }^{*} p \leq 0.05$.

\section{Theoretical Contributions}

Research on linkages between big five personality domains and five dimensions of social well-being conducted in China will likely contribute to the extant personality and well-being literature. First, this study provides empirical evidence about the relationship between big five personality and social wellbeing. The association between the big five personality and social well-being was evidenced in our study. However, our research also showed some inconsistencies with previous researches (Joshanloo et al., 2012). From our results, extraversion was 
significantly related to social integration; agreeableness was positively related to all five dimensions of social well-being; conscientiousness was positively related to social actualization, social coherence, and social contribution; neuroticism was negatively related to social integration, social acceptance, social actualization, and social coherence; openness was positively related to social integration, social acceptance, social coherence, and social contribution. This inconsistency may be explained by the fact that the differences between Iran and China. For instance, Iran is a non-Arab Muslim country; the interactions in Iran are regulated partly by religious norms (Joshanloo et al., 2012). In China, with the Reform and Opening, the way of thinking and behavior of Chinese are becoming more and more open and innovative (Ma, 2013). The goal of community construction in China is to establish the autonomous system of community residents (Fei, 2002). Community residents' committee is an important organization of residents' selfgoverning and self-service (Sun, 2016). Thus, most community residents can participate in community management and satisfy their own service needs via residents' committee, which will benefit residents' life quality.

Second, the study highlights the effect of social support on social well-being. The existing literature has shown the relationship between social support and subjective well-being or psychological well-being (Jasinskaja-Lahti et al., 2006; Brannan et al., 2013). Further, our study demonstrated social support is positively related to social well-being. Well-being is increasingly being associated with social and cultural relationships (Helliwell and Putnam, 2004). Community in China is increasingly becoming a place for residents to integrate into urban society (Chen et al., 2020). One of the most important responsibilities of the community is to achieve the society reconstruction (Fei, 2002). Thus, during the development of community, the Chinese government was committed to improving the quality of community services, which may provide more opportunities for residents to get more social support. Individuals having high social support means they had selected and built large and effective social networks, which can help to overcome difficulties in lives. With the help from their social relations, they will give a high appraisal to their circumstances and functioning in society; their social well-being also increases.

Third, the mediating effects were found for social support for relation between extraversion/agreeableness/ conscientiousness/neuroticism/openness and social well-being. This may contribute to the literature on the relationship between big five personality and social well-being (Hill et al., 2012; Joshanloo et al., 2012). Previous studies neglected to examine the relationship and the mechanism between big five personality and social well-being from the perspective of the community. Community is an important place for residents' daily activities. Individuals with different personality traits may build their social relations in different ways. Friends or family or neighbors around them may behave with different reactions. The different levels of social support will influence their evaluation of the social world, which may cause different levels of social well-being.

\section{Practical Implications}

Our study provides valuable insight into how individuals of different traits to improve their social well-being. Social support serves as a mediator in the relationship between big five personality and social well-being. The results also affirm the importance of social support that can enhance social well-being. When one's psychological, social, and/or resource needs are met, one is likely to experience greater social support, which is important for their well-being. Therefore, it is possible for residents to promote social support. Individuals should spend more time participating in community public affairs or other social activities that could offer opportunities for them to establish meaningful relationship with neighbors or friends.

\section{Limitations and Future Research}

Despite these findings, our research is not without limitations. First, culture is an important factor that can influence both personality traits and well-being (Diener et al., 2003; Hofstede and McCrae, 2004). Our study just discussed the mediating effect of social support between personality and social well-being. Future research should explore the effects of different cultural variables (such as power distance, collectivism/individualism etc.). In addition, comparative studies among different countries or regions are needed. Second, the cross-sectional design means that no causal conclusions for the found relationship can be made. Consequently, future researches should adopt longitudinal or experimental design to ascertain the relationship. Third, social support has usually been classified into several specific forms, such as informational support, emotional support, perceived social support (Taylor, 2011). In current study, we just regarded perceived social support as the mediating variable. So, future research should examine the effects of different forms of social support.

\section{Conclusion}

The research used a sample drawn from 1,658 Chinese residents to investigate the relationship between big five personality and social well-being and the mediating effect of social support in the relationship between big five personality and social well-being. Results of this study support previous studies that highlighted the relationship between big five personality and social support (Swickert et al., 2010; Barańczuk, 2019). In addition, this study demonstrated the effects of five personality traits on five dimensions of social well-being. Lastly, the results demonstrated the mediating role of social support in the associations between extraversion/agreeableness/conscientiousness/neuroticism/open ness and social well-being, respectively.

\section{DATA AVAILABILITY STATEMENT}

The raw data supporting the conclusions of this article will be made available by the authors, without undue reservation. 


\section{ETHICS STATEMENT}

The studies involving human participants were reviewed and approved by Yunnan University of Finance and Economics. Written informed consent for participation was not required for this study in accordance with the national legislation and the institutional requirements.

\section{AUTHOR CONTRIBUTIONS}

YY, YZ, and JL designed the research and wrote the manuscript. All authors planned and conducted the data collection. YZ, JZ, and DL analyzed the data and revised the

\section{REFERENCES}

Albanesi, C., Cicognani, E., and Zani, B. (2010). Sense of community, civic engagement and social well-being in italian adolescents. J. Commun. Appl. Soc. Psychol. 17, 387-406. doi: 10.1002/casp.903

Ayub, N. (2015). Predicting suicide ideation through intrapersonal and interpersonal factors: the interplay of Big-Five personality traits and social support. Pers. Ment. Health 9, 308-318. doi: 10.1002/pmh. 1301

Barańczuk, U. (2019). The five factor model of personality and social support: a meta-analysis. J. Res. Pers. 81, 38-46. doi: 10.1016/j.jrp.2019.05.002

Barrick, M. R., Mount, M. K., and Judge, T. A. (2001). Personality and performance at the beginning of the new millennium: what do we know and where do we go next? Intern. J. Select. Assess. 9, 9-30. doi: 10.1111/1468-2389. 00160

Brannan, D., Biswas-Diener, R., Mohr, C. D., Mortazavi, S., and Stein, N. (2013). Friends and family: a cross-cultural investigation of social support and subjective well-being among college students. J. Posit. Psychol. 8, 65-75. doi: 10.1080/17439760.2012.743573

Brislin, R. (1970). Back-translation for cross-cultural research. J. Cross Cult. Psychol. 1, 185-216. doi: 10.1177/135910457000100301

Chen, L. H. (2013). Gratitude and adolescent Athletes' well-being: the multiple mediating roles of perceived social support from coaches and teammates. Soc. Indic. Res. 114, 273-285. doi: 10.1007/s11205-012-0145-2

Chen, Z., Liu, S., Yu, Y., Bwanali, T. R., and Douangdara, V. (2020). Community satisfaction, sense of community, and social well-being in China. Soc. Behav. Pers. Intern. J. 48:e8648.

Chen, Z. X., and Li, Q. M. (2014). Verification of integrated model of well-being in different age and gender groups. Chin. J. Clin. Psychol. 5, 78-83.

Chen, Z. X., and Yu, Y. H. (2019). Urban social management, social support and social well-being: an empirical study of 2284 Chinese in 41 Cities, Northwest population. Ann. Rev. Psychol. 40, 44-56.

Cheung, F. M. (2004). Use of Western-and indigenously-developed personality tests in Asia. Appl. Psychol. Intern. Rev. 53, 173-191. doi: 10.1111/j.1464-0597. 2004.00167.x

Cheung, F. M., Leung, K., Zhang, J. X., Sun, H. F., Gan, Y. Q., Song, W. Z., et al. (2001). Indigenous Chinese personality constructs: is the fivefactor model complete? J. Cross Cult. Psychol. 32, 407-433. doi: 10.1177/ 0022022101032004003

Cobb, S. (1976). Social support as a moderator of life stress. Psychosom. Med. 38, 300-314. doi: 10.1097/00006842-197609000-00003

Cohen, S. (2004). Social relationships and health. Am. Psychol. 59, 676-684.

Cohen, S., and Syme, S. L. (1985). "Issues in the study and application of social support," in Social Support and Health, eds S. Cohen and S. L. Syme (Orlando, FL: Academic Press), 3-22.

Cohen, S., and Wills, T. A. (1985). Stress, social support and the buffering hypothesis. Psychol. Bull. 98, 310-357. doi: 10.1037/0033-2909.98.2.310

Cox, E. (2000). Creating a more civil society: community level indicators of social capital. Just Policy J. Austr. Soc. Policy 19, 100-107. manuscript. All authors listed have made direct and intellectual contribution to the article and approved the final version for publication.

\section{FUNDING}

This study was supported by the Chinese National Natural Science Fund (72064042), the Post-project of Chinese Ministry of Education (18JHQ080), the Philosophy and Social Science Research Project in Yunnan Province (QN202026), and the Science Research Fund of Yunnan Provincial Department of Education (2020J0384).

DeNeve, K. M., and Cooper, H. (1998). The happy personality: a meta-analysis of 137 personality traits and subjective well-being. Psychol. Bull. 124, 197-229. doi: 10.1037/0033-2909.124.2.197

Diener, E., and Diener, M. (1995). Cross-cultural correlates of life satisfaction and self-esteem. J. Pers. Soc. Psychol. 68, 653-663. doi: 10.1037/0022-3514.68.4.653

Diener, E., Oishi, S., and Lucas, R. E. (2003). Personality, culture, and subjective well-being: emotional and cognitive evaluations of life. Ann. Rev. Psychol. 54, 403-425. doi: 10.1146/annurev.psych.54.101601.145056

Fei, X. T. (2002). Autonomy of resident: new target of community construction in urban China. Jianghai Acad. J. 3, 15-18.

Funder, D. C., and Fast, L. A. (2010). "Personality in social psychology," in Handbook of Social Psychology, 5th Edn, eds S. T. Fiske, D. T. Gilbert, and G. Lindsey (New York, NY: Wiley), 668-697.

Gutiérrez, J. L. G., Jiménez, B. M., Hernández, E. G., and Pcn, C. (2005). Personality and subjective well-being: big five correlates and demographic variables. Pers. Individ. Differ. 38, 1561-1569. doi: 10.1016/j.paid.2004.09.015

Ha, S. E., and Kim, S. (2013). Personality and subjective well-being: evidence from South Korea. Soc. Indic. Res. 111, 341-359. doi: 10.1007/s11205-012-0009-9

Hartley, C., and Coffee, P. (2019). Perceived and received dimensional support: main and stress-buffering effects on dimensions of burnout. Front. Psychol. 10:1724. doi: 10.3389/fpsyg.2019.01724

Hayes, A. (2013). Introduction to Mediation, Moderation, and Conditional Process Analysis. New York, NY: Guilford.

Hayes, N., and Joseph, S. (2003). Big 5 correlates of three measures of subjective well-being. Pers. Individ. Differ. 34, 723-727. doi: 10.1016/s0191-8869(02) 00057-0

Helliwell, J. F., and Putnam, R. D. (2004). The social context of well-being. Philos. Trans. Biol. Sci. 359, 1435-1446.

Hill, P. L., Turiano, N. A., Mroczek, D. K., and Roberts, B. W. (2012). Examining concurrent and longitudinal relations between personality traits and social well-being in adulthood. Soc. Psychol. Pers. Sci. 3, 698-705. doi: 10.1177/ 1948550611433888

Hofstede, G., and McCrae, R. R. (2004). Personality and culture revisited: linking traits and dimensions of culture. Cross Cult. Res. 38, 52-88. doi: 10.1177/ 1069397103259443

Inoue, Y., Funk, D. C., Wann, D. L., Yoshida, M., and Nakazawa, M. (2015). Team identification and postdisaster social well-being: the mediating role of social support. Group Dyn. Theory Res. Pract. 19:31. doi: 10.1037/gdn0000019

Jasinskaja-Lahti, I., Liebkind, K., Jaakkola, M., and Reuter, A. (2006). Perceived discrimination, social support networks, and psychological well-being among three immigrant groups. J. Cross Cult. Psychol. 37, 293-311. doi: 10.1177/ 0022022106286925

John, O. P., Donahue, E. M., and Kentle, R. L. (1991). The 'Big Five' Inventory: Versions $4 a$ and 54. Technical Report. Berkeley, CA: Institute of Personality Assessment and Research.

John, O. P., and Srivastava, S. (1999). “The big five trait taxonomy: history, measurement, and theoretical perspectives," in Handbook of Personality: Theory and Research, eds L. A. Pervin and O. P. John (New York, NY: Guilford Press), $102-138$. 
Joshanloo, M., Rastegar, P., and Bakhshi, A. (2012). The Big Five personality domains as predictors of social wellbeing in Iranian university students. J. Soc. Pers. Relat. 29, 639-660. doi: 10.1177/0265407512443432

Keyes, C. L. M. (1998). Social well-being. Soc. Psychol. Q. 61, 121-140.

Keyes, C. L. M. (2002). The mental health continuum: from languishing to flourishing in life. J. Health Soc. Behav. 43, 207-222. doi: 10.2307/309 0197

Keyes, C. L. M. (2005). Mental health and/or mental illness? Investigating axioms of the complete state model of health. J. Consult. Clin. Psychol. 73, 539-548. doi: 10.1037/0022-006x.73.3.539

Keyes, C. L. M., and Ryff, C. D. (1998). "Generativity in adult lives: social structural contours and quality of life consequences," in Generativity and Adult Development: Perspectives on Caring for and Contributing to the Next Generation, eds D. McAdams and E. de St. Aubin (Washington, DC: American Psychological Association), 227-263. doi: 10.1037/10288-007

Keyes, C. L. M., and Shapiro, A. D. (2004). "Social well-being in the United States: a descriptive epidemiology," in How Healthy are We? A National Study of WellBeing at Midlife, eds O. G. Brim, C. D. Ryff, and R. C. Kessler (Chicago, IL: University of Chicago Press), 350-372.

Lee, R. M., Draper, M., and Lee, S. (2001). Social connectedness, dysfunctional interpersonal behaviors, and psychological distress: testing a mediator model. J. Counsel. Psychol. 48, 310-318. doi: 10.1037/0022-0167.48.3.310

Li, Q. M., and Chen, Z. X. (2015). Development level of big five personality in five dimensions and ten aspects: based on cross-sectional sample survey of 15-75 years old in China. Psychol. Sci. 1, 131-138.

Ma, X. (2013). The thinking way of Chinese and its transformation of modernity. J. Liaoning Administ. Coll. 15, 157-158.

Miao, Y. J., and Wang, Q. H. (2009). Investigation and Research on College Students' social well-being. J. Gannan Norm. Univer. 30, 76-81.

Minkov, M., Fons, J. R., van de Vijver, O., and Michael, S. (2019). A test of a new short big-five tool in large probabilistic samples from 19 countries. Pers. Individ. Differ. 151:109519. doi: 10.1016/j.paid.2019.109519

Oh, H. J., Ozkaya, E., and LaRose, R. (2014). How does online social networking enhance life satisfaction? The relationships among online supportive interaction, affect, perceived social support, sense of community, and life satisfaction. Comput. Hum. Behav. 30, 69-78. doi: 10.1016/j.chb.2013. 07.053

Podsakoff, P. M., MacKenzie, S. B., Lee, J. Y., and Podsakoff, N. P. (2003). Common method biases in behavioral research: a critical review of the literature and recommended remedies. J. Appl. Psychol. 88, 879-903. doi: 10.1037/0021-9010. 88.5.879

Preacher, K. J., and Hayes, A. F. (2004). SPSS and SAS procedures for estimating indirect effects in simple mediation models. Behav. Res. Methods Instr. Comput. 36, 717-731. doi: 10.3758/bf03206553
Rhee, J., Seog, S. D., Bozorov, F., and Dedahanov, A. T. (2017). Organizational structure and employees' innovative behavior: the mediating role of empowerment. Soc. Behav. Pers. Intern. J. 45, 1523-1536. doi: 10.2224/sbp.6433

Sarason, B. R., Sarason, I. G., and Pierce, G. R. (1990). “Traditional views of social support and their impact on assessment," in Social Support: An Interactional View, eds B. R. Sarason, I. G. Sarason, and G. R. Pierce (New York, NY: Wiley), 9-25. doi: 10.1080/07349165.1982.9725924

Siedlecki, K. L., Salthouse, T. A., Oishi, S., and Jeswani, S. (2014). The relationship between social support and subjective well-being across age. Soc. Indic. Res. 117, 561-576. doi: 10.1007/s11205-013-0361-4

Sohi, K. K., Singh, P., and Bopanna, K. (2017). Ritual participation, sense of community, and social well-being: a study of seva in the sikh community. J. Relig. Health 57, 2066-2078. doi: 10.1007/s10943-017-0424-y

Sun, B. Y. (2016). Why is it possible for the urban community residents' committees to "de-administrate"? Nanjing Soc. Sci. 7, 51-58.

Swickert, R. J., Hittner, J. B., and Foster, A. (2010). Big Five traits interact to predict perceived social support. Pers. Individ. Differ. 48, 736-741. doi: 10.1016/j.paid. 2010.01.018

Taylor, S. E. (2011). "Social support: a review," in Oxford Library of Psychology. The Oxford Handbook of Health Psychology, ed. H. S. Friedman (New York, NY: Oxford University Press), 189-214.

Udayar, S., Urbanaviciute, I., and Rossier, J. (2018). Perceived social support and Big Five personality traits in middle adulthood: a 4-year cross-lagged path analysis. Appl. Res. Q. Life 15, 395-414. doi: 10.1007/s11482-018-969 4-0

Wong, S. T., Yoo, G. J., and Stewart, A. L. (2007). An empirical evaluation of social support and psychological well-being in older Chinese and Korean immigrants. Ethn. Health 12, 43-67. doi: 10.1080/13557850600824104

Zhang, W., Chen, Q., McCubbin, H., McCubbin, L., and Foley, S. (2011). Predictors of mental and physical health: individual and neighborhood levels of education, social well-being, and ethnicity. Health Place 17, 238-247. doi: 10.1016/j. healthplace.2010.10.008

Zhou, J. (2010). Structural validity analysis of Big Five Personality Inventory (BFI). Manag. Observ. 30, 252-253.

Conflict of Interest: The authors declare that the research was conducted in the absence of any commercial or financial relationships that could be construed as a potential conflict of interest.

Copyright (c) $2021 \mathrm{Yu}$, Zhao, Li, Zhang and Li. This is an open-access article distributed under the terms of the Creative Commons Attribution License (CC BY). The use, distribution or reproduction in other forums is permitted, provided the original author(s) and the copyright owner(s) are credited and that the original publication in this journal is cited, in accordance with accepted academic practice. No use, distribution or reproduction is permitted which does not comply with these terms. 